\title{
Transcriptomic analysis of aging mouse sciatic nerve reveals early pathways leading to sarcopenia
}

Nicole Comfort $^{1 *}$, Meethila Gade ${ }^{1}$, Madeleine Strait ${ }^{1}$, Samantha J. Merwin ${ }^{1}$, Daphne Antoniou ${ }^{2}$, Anna Memou ${ }^{3}$, Hardy J. Rideout ${ }^{3}$, Stefania Corti ${ }^{4}$, Shingo Kariya ${ }^{5}$, Diane B. Re ${ }^{1,6,7^{*}}$

${ }^{1}$ Department of Environmental Health Sciences, Mailman School of Public Health, Columbia University, New York, NY, USA

${ }^{2}$ Center for Basic Research, Biomedical Research Foundation of the Academy of Athens, Athens, Greece

${ }^{3}$ Center for Clinical, Experimental Surgery, and Translational Research, Biomedical Research

Foundation of the Academy of Athens, Athens, Greece

${ }^{4}$ Neuroscience Section, Dino Ferrari Centre, Department of Pathophysiology and

Transplantation, University of Milan, Milan, Italy

${ }^{5}$ Department of Neurology, Vagelos College of Physicians and Surgeons, Columbia University, New York, NY, USA

${ }^{6}$ Center for Motor Neuron Biology and Disease, Columbia University, New York, NY, USA

${ }^{7}$ NIEHS Center for Environmental Health Sciences in Northern Manhattan, Columbia

University, New York, NY, USA

*Corresponding authors’ emails: dr2240@cumc.columbia.edu; nicole.comfort@columbia.edu 


\begin{abstract}
:
Background: Sarcopenia, the age-associated decline in skeletal muscle mass and strength, has long been considered a disease of muscle only, but accumulating evidence suggests that sarcopenia could originate from the neural components controlling muscles. To identify early molecular changes in the efferent nerves that may drive sarcopenia initiation, we performed a longitudinal transcriptomic analysis of the sciatic nerve in aging mice.
\end{abstract}

Methods: Sciatic nerve and gastrocnemius muscle were obtained from young adult, middleaged, old, and sarcopenic $(5,18,21$ and 24 months old, respectively) C57BL/6J female mice ( $\mathrm{n}=6$ per age group). Sciatic nerve RNA was extracted and subjected to RNA sequencing (RNA-seq), with real-time quantitative reverse transcription PCR (qRT-PCR) validation of differentially expressed genes (DEGs). Functional enrichment analysis of clusters of genes associated with patterns of gene expression across age groups was performed. Sarcopenia was confirmed with qRT-PCR of previously established markers of sarcopenia onset in gastrocnemius muscle.

Results: We detected 33 significant DEGs in sciatic nerve of 18-month-old mice compared to 5month-old mice (absolute value of fold change $>2$; false discovery rate $[$ FDR $]<0.05$ ) which we validated with qRT-PCR of the three top up- and down-regulated genes. Up-regulated genes were associated with circadian rhythm and the AMPK signaling pathway, while down-regulated genes were associated with biosynthesis and metabolic pathways and circadian rhythm.

Strikingly, we detected a significant increase in Myog expression $\left(\log _{2}\right.$ fold change $=18.93$, FDR $q$-value $=1.54 \times 10^{-12}$ ) in sciatic nerve of 18 -month-old mice, before up-regulation in muscle was observed. We identified seven clusters of genes with similar expression patterns across groups. Functional enrichment analysis of these clusters revealed biological processes that may be implicated in sarcopenia initiation including extracellular matrix organization and circadian regulation of gene expression.

Conclusions: Gene expression changes in mouse peripheral nerve can be detected prior to overt clinical onset of sarcopenia. These early molecular changes we report shed a new light on biological processes that may be implicated in sarcopenia initiation and pathogenesis. Future 
studies will validate which of the key changes we reported have disease modifying and/or biomarker potential.

Keywords: Aging, sarcopenia, skeletal muscle, RNA-seq, motor unit, frailty

\section{Introduction}

Based on its symptomatic and histological features, sarcopenia, the age-associated loss in skeletal muscle mass and strength, has long been considered a disease of skeletal muscle fibers only. However, accumulating evidence suggests that sarcopenia could originate from the neural components controlling muscle strength and hence be defined as a neuromuscular disease (Krishnan et al. 2016; Pannérec et al. 2016; Rolland et al. 2008; Ryall, Schertzer, and Lynch 2008). Although current evidence suggests that motor nerves and presynapses of neuromuscular junctions (NMJs) could be implicated in sarcopenia pathophysiology, their role in initiation of sarcopenia has not been fully elucidated.

We hypothesized that sarcopenia onset and its early molecular programming could be captured in the major nerve of the lower limb, the sciatic nerve, reflected by changes in gene expression in nerves preceding sarcopenia development in muscle. Most previous gene expression profiling studies investigating the mechanisms of sarcopenia pathophysiology have been limited by their use of microarrays for peripheral nerve molecular profiling. Microarrays cover only a defined set of transcripts, have high background levels due to cross-hybridization, and have a limited dynamic range due to both background as well as saturation of signals. However, studies that utilized next-generation sequencing technologies have so far focused on age-related changes in muscles rather than nerves (Zhou et al. 2018).

To circumvent these issues and capture early molecular events responsible for the initiation of sarcopenia, we performed deep RNA sequencing (RNA-seq) (Wang, Gerstein, and Snyder 2009) of sciatic nerves, isolated at different time points from healthy young (5 months), middle-aged (18 and 21 months), and sarcopenic (24 months) old mice. Our objective was to identify early changes in gene expression related to the development of sarcopenia via transcriptomic profiling of aging sciatic nerves in vivo. In agreement with a potential neurogenic origin of sarcopenia, we identified a series of relevant gene expression changes in mouse peripheral nerve prior to overt clinical onset of sarcopenia in muscles. Among the early 
molecular events that we report at 18 months, we confirm the importance of biological processes previously linked to sarcopenia while also reporting new findings that shed light on other pathophysiological mechanisms that may be implicated in sarcopenia initiation and pathogenesis. To our knowledge, this study is the first to utilize untargeted RNA-seq to investigate the transcriptome of sciatic nerves in the context of sarcopenia. Future studies are warranted to confirm whether neuromuscular biomarkers of sarcopenia could be identified in efferent nerves at even earlier timepoints and to validate which among them are phenotypic drivers with potential implications for sarcopenia prevention and therapy.

\section{Methods}

2.1. Mice and sample collection

Female C57BL/6J mice were obtained from the National Institute on Aging mouse colony, two weeks before turning 5, 18, 21, and 24 months old. The mice were kept in the Columbia University Medical Center animal facility for two weeks and then were used immediately. The mice had free access to the standard pellet and water diet, and were maintained under a constant 12 -hour light/dark cycle at an environmental temperature of $21-23{ }^{\circ} \mathrm{C}$. All animal procedures complied with the Guide for the Care and Use of Laboratory Animals and were approved by the Columbia University Institutional Animal Care and Use Committee (Approval ID: AC-AAAN8900).

At 5 (young adult negative control), 18 (middle-aged), 21 (old), and 24 (sarcopenic positive control) months, mice ( $n=6$ per group) were euthanized via deep anesthesia followed by decapitation. Sciatic nerves were quickly dissected before undergoing RNA extraction. The remaining leg was snap-frozen in liquid nitrogen and stored at $-80^{\circ} \mathrm{C}$.

\subsection{RNA extraction}

\subsubsection{Sciatic nerve}

Total RNA was isolated from freshly dissected sciatic nerves using the Invitrogen PureLink ${ }^{\text {TM }}$ RNA Mini Kit (Cat \# 12183018A) following the manufacturer's protocol for $\leq 10$ mg fresh soft animal tissue. All samples had total RNA concentration and RNA integrity numbers (RINs) assessed prior to sequencing or real-time quantitative reverse transcription PCR 
(qRT-PCR). RINs were evaluated using an Agilent 2100 Bioanalyzer and ranged from 6.2 to 9.6 (24 samples; average: 8.7, Supplemental Table 1).

\subsubsection{Gastrocnemius muscle}

The gastrocnemius muscles were dissected from the leg on dry ice and $50 \mathrm{mg}$ of tissue was flash frozen in duplicate. The tissue samples were then placed in a pre-chilled Eppendorf tube and homogenized with a Qiagen TissueRuptor II (Cat \# 9002755) with disposable probes (Qiagen, Cat \# 990890). Total RNA extraction was performed using trizol-chloroform extraction followed by column centrifugation using the Invitrogen PureLink ${ }^{\mathrm{TM}}$ RNA Mini Kit following the manufacturer's instructions. Then, samples were treated with DNAse followed by wash buffer and eluted in water. Total RNA was quantified using the RiboGreen ${ }^{\mathrm{TM}}$ RNA Quantitation Kit (Thermo Fisher, Cat \# R11490).

\subsection{Library preparation and RNA sequencing}

Libraries were constructed at the Columbia Biomedical Core Facility. Briefly, mRNA was enriched from total RNA via poly-A pull-down using the Illumina TruSeq Stranded mRNA kit according to the manufacturer's instructions. Libraries were 2x100 base-pair (bp) paired-end sequenced for each sample on an Illumina HiSeq4000 using 75-cycle High Output Kits (target 60 million reads per sample) at the Columbia University Genome Center. Raw sequencing data and processed data will be deposited at the NIH Gene Expression Omnibus (GEO).

\subsection{RNA sequencing analysis, quality control, and identification of differentially expressed genes}

We used RTA (Illumina) for base calling and bcl2fastq2 (version 2.17) for converting BCL to fastq format, coupled with adaptor trimming. Quality control of the raw and trimmed reads was performed using FastQC and MultiQC (Andrews 2010; Ewels et al. 2016). We aligned trimmed reads that passed quality control (Phred score $>30$ ) to the mouse reference genome and transcriptome annotation (mm10, UCSC) using STAR aligner (version 2.5.2b) (Dobin and Gingeras 2015) and quantified the reads that aligned uniquely to the transcriptome using featureCounts (v1.5.0-p3) (Liao, Smyth, and Shi 2014). Sequencing yielded libraries with an average size of 39 million reads after alignment. 
We used the DESeq2 (version 1.34.0) package for R (version 4.1.1; R-project.org/) (Love, Huber, and Anders 2014) for further quality control, exploratory data anslysis, and differential expression analysis. For sample-level quality control, we conducted principal component analysis (PCA) of the RNA-seq data by first normalizing the counts for each sample using size factors obtained from DESeq2, log-transforming the normalized counts (using the regularized-logarithm [rlog] transformation), and computing the principal components of the resulting matrix using the plotPCA function of DESeq2 (Supplemental Figure 1A). We also created heatmaps of the correlation of rlog-transformed gene expression for all pairwise combinations of samples (Supplemental Figure 1B). Based on this quality control assessment, we removed two samples that were outliers from downstream analyses (resulting PCA plot and correlation heatmap of rlog-transformed gene expression shown for $N=22$ shown in

\section{Supplemental Figure 2).}

We removed 4,749 (20.3\%) rows of the DESeqDataSet that had no counts or only a single count across all included samples $(N=22)$. We additionally specified that at least $20 \%$ of samples (i.e., at least 5 samples) have a count of 10 or higher, resulting in removal of an additional 3,079 genes (13.1\%). In total, 7,828 (33.4\%) of 23,416 unique genes were filtered out, resulting in 15,588 genes analyzed in the DESeqDataSet. DESeq2 fits negative binomial generalized linear models for each gene and determines differential expression for pairwise comparisons by applying the Wald test for significance testing. Adjusted $p$-values were calculated by Benjamini-Hochberg false discovery rate (FDR). We considered genes that had an absolute value of a $\log _{2}$ FoldChange (LFC) $>1.0$ with adjusted p-value $<0.05$ to be differentially expressed genes (DEGs).

\subsection{Validation of differentially expressed genes with qRT-PCR}

\subsubsection{SYBR ${ }^{\circ}$ Green RT-PCR}

For select genes in sciatic nerve identified as differentially expressed by DESeq2, we validated the RNA-seq results using qRT-PCR. We also performed qRT-PCR of the genes Chrnd, Gadd45a, Myog, Runxl, Chrng, and Ncam in gastrocnemius muscle to confirm onset of sarcopenia. For qRT-PCR, Qiagen QuantiTect Primers were reconstituted in TE buffer (pH 8.0). Primer details are listed in Supplemental Table 2. The reverse transcription reaction was performed using the Promega GoScript ${ }^{\mathrm{TM}}$ Reverse Transcriptase kit (Cat \# PRA5000) for a 
reaction volume of $20 \mu \mathrm{L}$. This was followed by SYBR ${ }^{\circledR}$ Green RT-qPCR reaction using KiCqStart $^{\mathrm{TM}} \mathrm{SYBR}^{\circledR}$ Green qPCR ReadyMix ${ }^{\mathrm{TM}}$ (Millipore Sigma, Cat \# KCQS00). Briefly, 5 $\mu \mathrm{L}$ reaction mix was prepared using $2.5 \mu \mathrm{L}$ qPCR ready mix, $1.25 \mu \mathrm{L}$ sample cDNA, and 1.25

$\mu \mathrm{L}$ primers. The RT-PCR reaction was run on Bio-Rad CFX384 Touch Real-Time PCR System as per kit instructions for three technical replicates.

\subsection{2. qRT-PCR data analysis}

RT-PCR results were analyzed in RStudio (version 3.6.0). Data was cleaned by excluding samples with cycle threshold $(\mathrm{Ct})$ values $>35$ and only $1 \mathrm{read} / \mathrm{technical}$ replicate. Raw $\mathrm{Ct}$ values were then normalized to the geometric mean of housekeeping genes (Ppia and Hprt,

Supplemental Figure 3). The resulting delta $\mathrm{Ct}(\mathrm{dCt})$ values were further normalized to the 5month age group, which served as the reference, to create delta-delta $\mathrm{Ct}$ values (ddCT). Plots were generated using GraphPad Prism (version 8.4.0). Only the raw dCT values were plotted for assays (Chrng, Myog, Mstn) with many non-detects in the reference group. A one-way ANOVA was used to test for any statistically significant differences between the groups followed by post hoc analysis using Fisher's Least Significant Difference (LSD) test. We report significant differences in ddCT comparing each group (18, 21, 24 months) to the reference group (5 months).

\subsection{Pattern identification}

We analyzed all pair-wise comparisons simultaneously using the Likelihood Ratio Test (LRT) in DESeq2 to identify any genes that showed an interesting change in expression across the different groups $(5,18,21,24$ months). We subset the rlog-transformed normalized counts to contain only genes significant with adjusted $p$-value $<0.05$ and explored clusters of genes with similar expression patterns across sample groups using the 'degPatterns' function from the DEGreport R package. Gene lists associated with each identified cluster were used as input for functional enrichment analysis.

\subsection{Functional and pathway enrichment analysis}

DEGs identified by the Wald test were explored using gene ontology (GO) functional enrichment analysis and KEGG (Kyoto Encyclopedia of Genes and Genomes) pathway 
enrichment analysis performed using the database for annotation, visualization and integrated discovery (DAVID) (https://david.ncifcrf.gov/) (Sherman et al. 2007; Dennis et al. 2003; Huang et al. 2007; Huang, Sherman, and Lempicki 2009). Pathways or GO terms were considered significant at $p$-values $<0.05$.

To investigate the biological relevance of clusters of DEGs associated with main expression patterns, we performed GO functional enrichment analysis using GOrilla, a publicly available GO enrichment tool (http://cbl-gorilla.cs.technion.ac.il) (Eden et al. 2009). GOrilla compares a target gene list (in this case, genes assigned to a given cluster) to a background set of genes $(N=2,925$ genes significant in the LRT with FDR $<0.05)$ and assesses the significance of enrichment for GO terms using the hypergeometric test.

\section{Results}

3.1. Confirmation of sarcopenia onset in C57BL/6J mice via qRT-PCR of genes in gastrocnemius muscle

To validate the onset of overt sarcopenia in our different aged mouse groups in an unbiased manner (independent of mouse size/weight/basal activity/hydration, etc.), we performed qRT-PCR quantification of the following genes, associated with NMJ denervation, in gastrocnemius muscle: nicotinic acetylcholine receptor (nAChR) gamma and delta subunits (Chrng and Chrnd), runt-related transcription factor-1 (Runxl), growth arrest and DNA damageinducible 45a (Gadd45a), and Myogenin (Myog). It has been previously established that increased expression of these genes in aging muscle is concomitant with the transition to sarcopenia in 24-month-old female C57BL/6J mice. As reported previously (Barns et al. 2014), we observed significantly increased expression in Chrnd, Chrng, Runxl, and Gadd45a at age 24 months (Supplemental Figure 4). Note that for Chrng, the substantial increase in expression measured at 24 months was not tested for statistical significance because of the very low expression (mostly non-detectable) in the reference group (5 m old). There was an increase in Myog expression at 24 months that was not statistically significant.

3.2. Identification of differentially expressed genes (DEGs) in sciatic nerve by RNA-seq The number of DEGs $(|\mathrm{LFC}|>1.0, \mathrm{FDR}<0.05)$ identified using DESeq2 is shown in

Table 1. The full DESeq2 results, including the list of these DEGs complete with their full gene 
name, associated LFC and $p$-values, are shown in Supplemental Spreadsheet 1. Because we were interested in the earliest transcriptional changes that may precede initiation of sarcopenia pathology, we assessed the contrast between 18-month-old and 5-month-old mice in more detail. Comparing these two groups, a total of 33 DEGs were identified, including 16 up-regulated and 17 down-regulated DEGs (Figure 1, Table 2). Interestingly, the most up-regulated gene (according to LFC) was Myog with a LFC $=18.93\left(\right.$ FDR $q$-value $\left.=1.54 \times 10^{-12}\right)$. Volcano plots of the other pairwise comparisons are shown in Supplemental Figure 5.

To explore the biological roles of up- and down-regulated DEGs, we performed functional and pathway enrichment analyses on the DEGs comparing 18-month-old and 5month-old mice (listed in Table 2) using DAVID. Up-regulated DEGs were associated with biological processes and KEGG pathways related to circadian rhythm $(p=0.02)$, circadian entrainment ( $p=0.06$ ), and the AMP-activated protein kinase (AMPK) signaling pathway $(p=0.08)$, while down-regulated DEGs were associated with various pathways related to biosynthetic processes, including sterol, cholesterol, steroid, and isoprenoid biosynthetic processes (Table 3, Supplemental Table 3). Circadian rhythm was also enriched $(p=0.04)$ among down-regulated genes including neuronal PAS domain protein 2 (Npas2) and the gene encoding Aryl hydrocarbon receptor nuclear translocator-like protein 1 (Arntl), which both displayed similar gene expression patterns across age groups (Supplemental Figure 6). 


\begin{tabular}{|c|c|c|}
\hline \multicolumn{3}{|c|}{ \# DEGs FDR $<\mathbf{0 . 0 5}$} \\
\hline Contrast & LFC $>\mathbf{1 . 0}$ & LFC $<-\mathbf{1 . 0}$ \\
\hline 18 vs 5 months & $16(0.1 \%)$ & $17(0.11 \%)$ \\
\hline 21 vs 5 months & $18(0.12 \%)$ & $10(0.064 \%)$ \\
\hline 24 vs 5 months & $50(0.32 \%)$ & $18(0.12 \%)$ \\
\hline 21 vs 18 months & $13(0.083 \%)$ & $5(0.032 \%)$ \\
\hline 24 vs 18 months & $30(0.19 \%)$ & $1(0.0064 \%)$ \\
\hline 24 vs 21 months & $3(0.019 \%)$ & $1(0.0064 \%)$ \\
\hline
\end{tabular}

Table 1. Number (\%) of differentially expressed genes (DEGs) out of 15,588 genes.

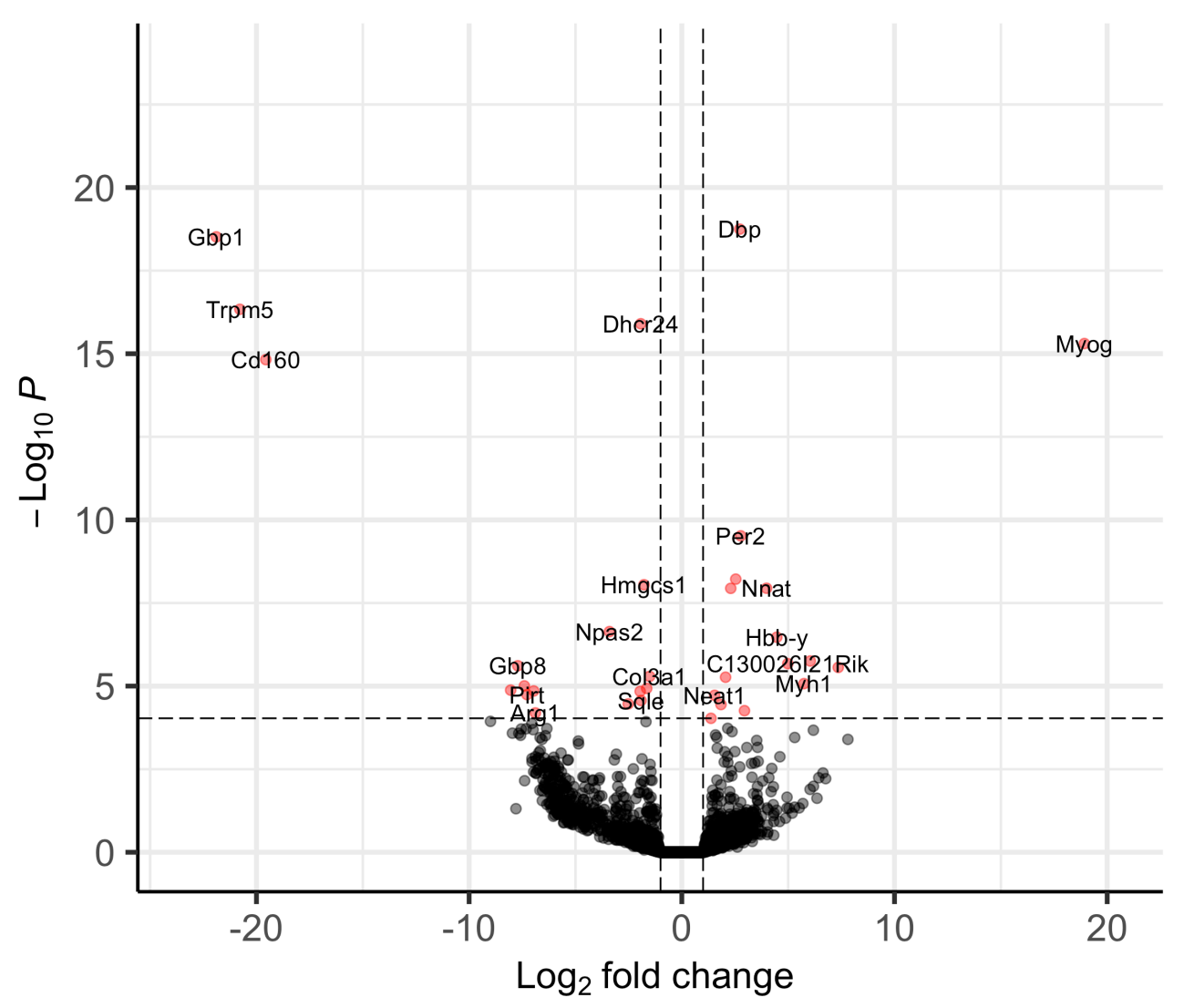

total $=15588$ variables

Figure 1. Volcano plot of differentially expressed genes (of 15,588 genes analyzed) in sciatic nerve comparing 18-month-old mice $(n=5)$ to 5 -month-old mice $(n=6)$. The dashed line is the FDR q-value $=0.05$. The 33 genes significant at $|\mathrm{LFC}|>1.0$ and FDR q-value $<0.05$ are plotted in red. 


\begin{tabular}{|c|c|c|c|}
\hline & Gene & $\log _{2}$ Fold Change & FDR $q$-value \\
\hline \multirow[t]{16}{*}{ Upregulated } & Myog & 18.93 & $1.54 \mathrm{E}-12$ \\
\hline & $\operatorname{Lmod} 2$ & 7.35 & $2.51 \mathrm{E}-03$ \\
\hline & $H b a-x$ & 6.04 & $1.96 \mathrm{E}-03$ \\
\hline & Myhl & 5.74 & $6.56 \mathrm{E}-03$ \\
\hline & C130026I21Rik & 4.98 & $2.19 \mathrm{E}-03$ \\
\hline & $H b b-y$ & 4.47 & $4.05 \mathrm{E}-04$ \\
\hline & Nnat & 3.98 & $1.62 \mathrm{E}-05$ \\
\hline & Lep & 2.95 & $2.76 \mathrm{E}-02$ \\
\hline & Per 2 & 2.77 & $6.72 \mathrm{E}-07$ \\
\hline & $D b p$ & 2.72 & $2.37 \mathrm{E}-15$ \\
\hline & Ppp2r2c & 2.54 & $1.18 \mathrm{E}-05$ \\
\hline & Col24al & 2.30 & $1.62 \mathrm{E}-05$ \\
\hline & Tmem $45 b$ & 2.06 & $4.42 \mathrm{E}-03$ \\
\hline & Per3 & 1.84 & $1.86 \mathrm{E}-02$ \\
\hline & Neat1 & 1.54 & $1.09 \mathrm{E}-02$ \\
\hline & Tef & 1.37 & $4.40 \mathrm{E}-02$ \\
\hline \multirow[t]{17}{*}{ Downregulated } & Col3al & -1.52 & $4.42 \mathrm{E}-03$ \\
\hline & $\mathrm{Hmgcr}$ & -1.65 & $8.32 \mathrm{E}-03$ \\
\hline & Hmgcs 1 & -1.78 & $1.54 \mathrm{E}-05$ \\
\hline & Sqle & -1.92 & $1.51 \mathrm{E}-02$ \\
\hline & Dhcr24 & -1.93 & $4.87 \mathrm{E}-13$ \\
\hline & Arntl & -1.96 & 8.97E-03 \\
\hline & $M v d$ & -2.52 & $1.84 \mathrm{E}-02$ \\
\hline & Npas 2 & -3.40 & $2.98 \mathrm{E}-04$ \\
\hline & Arg1 & -6.88 & $3.12 \mathrm{E}-02$ \\
\hline & $P 2 r x 1$ & -6.96 & 8.97E-03 \\
\hline & Pirt & -7.28 & $1.08 \mathrm{E}-02$ \\
\hline & Tnip3 & -7.40 & $7.32 \mathrm{E}-03$ \\
\hline & Gbp 8 & -7.71 & $2.38 \mathrm{E}-03$ \\
\hline & Adamts 16 & -8.05 & $8.96 \mathrm{E}-03$ \\
\hline & Cd160 & -19.56 & $3.90 \mathrm{E}-12$ \\
\hline & Trpm5 & -20.78 & $2.39 \mathrm{E}-13$ \\
\hline & $G b p 1$ & -21.89 & $2.37 \mathrm{E}-15$ \\
\hline
\end{tabular}

Table 2. The 33 DEGs identified in sciatic nerve comparing 18-month-old mice $(n=5)$ to healthy, young control mice 5 months old $(n=6)$, including 16 up-regulated genes and 17 down-regulated genes. 


\begin{tabular}{|c|c|c|c|c|c|}
\hline & KEGG Pathway & $\begin{array}{l}\text { Gene } \\
\text { count }\end{array}$ & $P$-value & $\begin{array}{l}\text { FDR } q- \\
\text { value }\end{array}$ & Genes \\
\hline \multirow[t]{4}{*}{ Upregulated } & Circadian rhythm & 2 & $2.00 \mathrm{E}-02$ & $4.60 \mathrm{E}-01$ & Per2, Per3 \\
\hline & Tight junction & 2 & $5.70 \mathrm{E}-02$ & 4.60E-01 & Myh1, Ppp2r2c \\
\hline & Circadian entrainment & 2 & $6.20 \mathrm{E}-02$ & 4.60E-01 & Per2, Per3 \\
\hline & $\begin{array}{l}\text { AMPK signaling } \\
\text { pathway }\end{array}$ & 2 & $8.10 \mathrm{E}-02$ & 4.60E-01 & Lep, $P p p 2 r 2 c$ \\
\hline \multirow[t]{5}{*}{ Downregulated } & $\begin{array}{l}\text { Biosynthesis of } \\
\text { antibiotics }\end{array}$ & 5 & $1.10 \mathrm{E}-04$ & $2.50 \mathrm{E}-03$ & $\begin{array}{l}\text { Hmgcr, Hmgcsl, Argl, Mvd, } \\
\text { Sqle }\end{array}$ \\
\hline & $\begin{array}{l}\text { Terpenoid backbone } \\
\text { biosynthesis }\end{array}$ & 3 & $3.80 \mathrm{E}-04$ & 4.40E-03 & $H m g c r, H m g c s 1, M v d$ \\
\hline & Metabolic pathways & 6 & $1.50 \mathrm{E}-02$ & $1.10 \mathrm{E}-01$ & $\begin{array}{l}\text { Dhcr24, Hmgcr, Hmgcs1, } \\
\text { Arg1, Mvd, Sqle }\end{array}$ \\
\hline & Steroid biosynthesis & 2 & $2.40 \mathrm{E}-02$ & $1.40 \mathrm{E}-01$ & Dhcr24, Sqle \\
\hline & Circadian rhythm & 2 & $4.00 \mathrm{E}-02$ & $1.80 \mathrm{E}-01$ & Arntl, Npas 2 \\
\hline
\end{tabular}

Table 3. KEGG pathway analysis of up- and down-regulated DEGs comparing 18month-old mice $(n=5)$ to 5 -month-old mice $(n=6)$. 


\subsection{Validation of DEGs in sciatic nerve via qRT-PCR}

We performed qRT-PCR on a set of DEGs to validate the RNA-seq results. As shown in

Figure 2, the gene expression in the sciatic nerve measured by qRT-PCR agreed with our RNAseq findings. Compared to 5-month-old mice, there was a significant increase in expression of period circadian regulator $2(\operatorname{Per} 2, p=0.0072)$, D site albumin promoter binding protein $(\mathrm{Dbp}$, $p=0.0039)$, and protein phosphatase 2 , regulatory subunit $\mathrm{B}$, gamma $(P p p 2 r 2 c, p=0.0004)$ (Figure 2A-C) in 18-month-old mice. We additionally confirmed that the genes guanylate binding protein 11 (Gbpl), 24-dehydrocholesterol reductase (Dhcr24), and 3-hydroxy-3methylglutaryl-Coenzyme A synthase 1 (Hmgcs 1) were significantly down-regulated in 18month-old mice compared to 5-month-old mice ( $p=0.0009$ for Gbpl and $p<0.0001$ for Dhcr24 and Hmgcs 1 ) (Figure 2D-F). We also confirmed the upregulation of Myog in 18-month-old mouse sciatic nerve compared to 5-month-old mice (Figure 3). Myog was not detected in sciatic nerve of 5-month-old mice. 
A
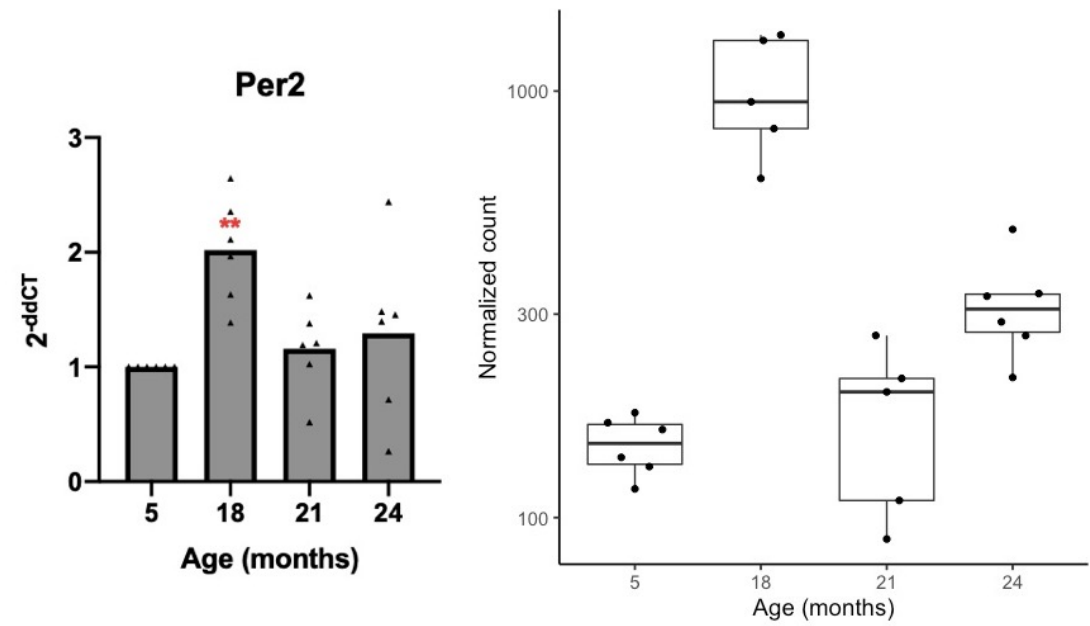

B
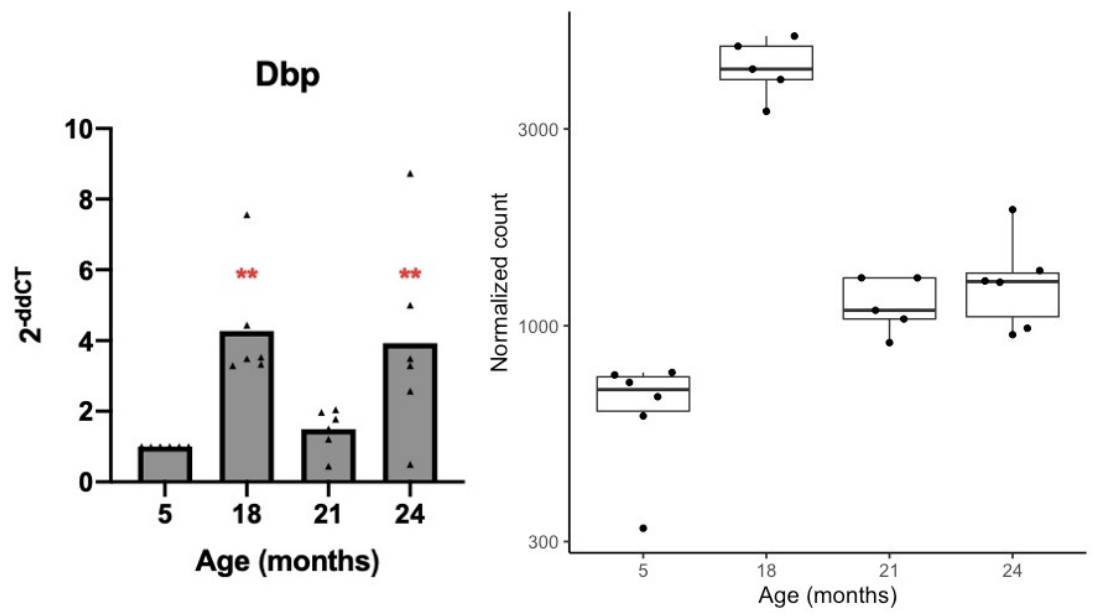

$\mathrm{C}$

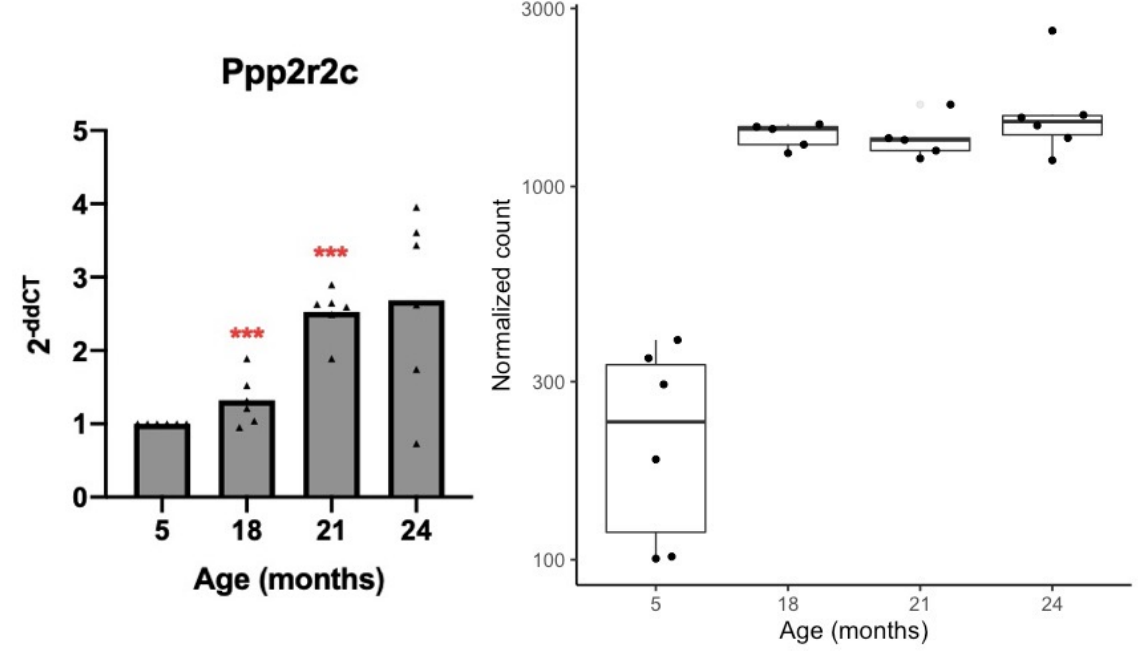


$\mathrm{D}$
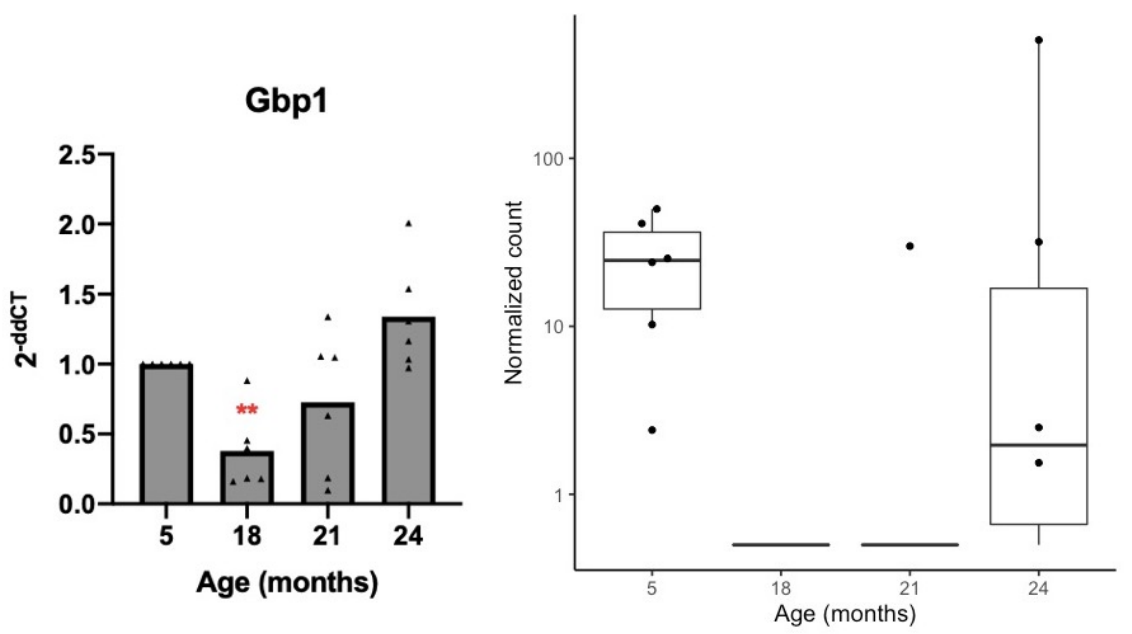

$\mathrm{E}$
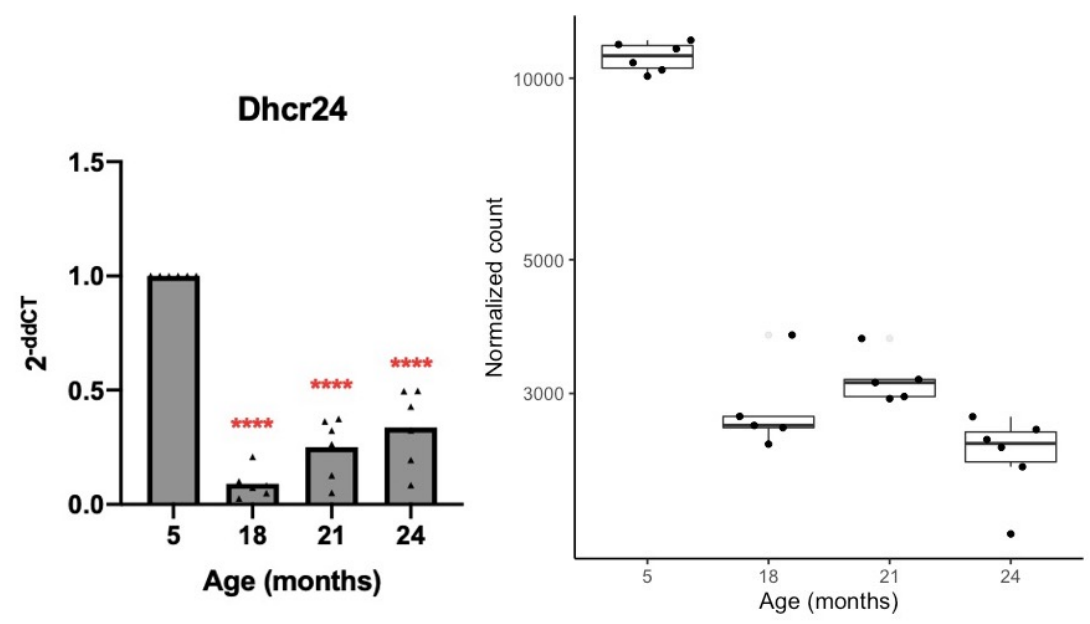

$\mathrm{F}$
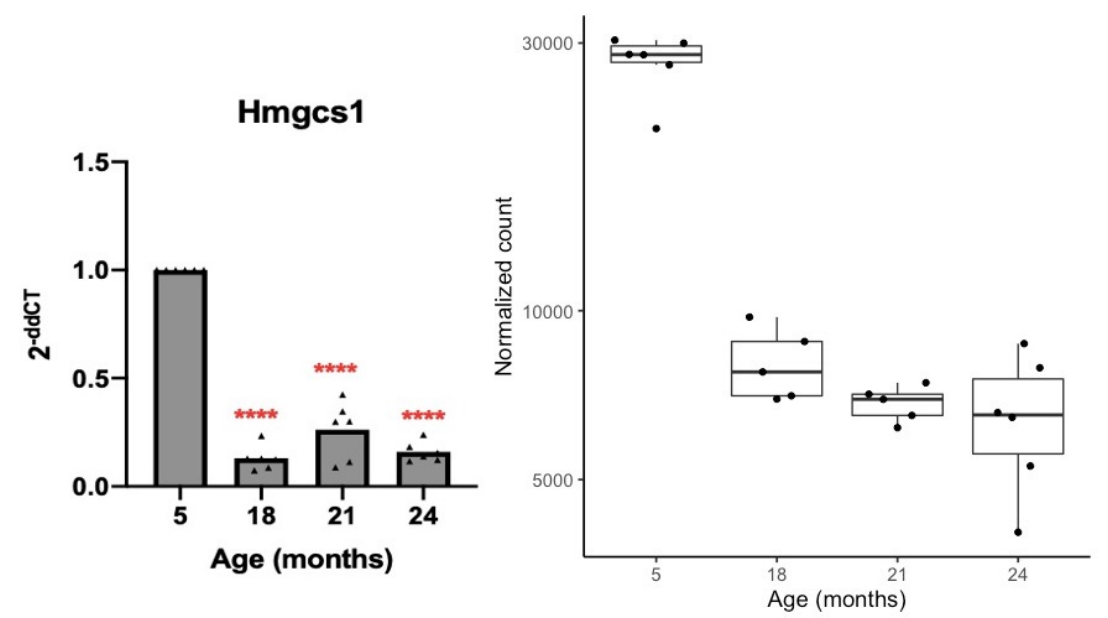

Figure 2. Gene expression of up-regulated (A-C) and down-regulated (D-F) genes in sciatic nerve of mice aged 5, 18, 21, and 24 months assessed by qRT-PCR and RNA-seq. Left: Relative gene expression assessed by qRT-PCR; Right: Corresponding plot of normalized counts from RNA-seq analysis ( $n=5-6$ per age group). For RT-PCR, the delta-delta $\mathrm{Ct}$ was calculated using 
Ppia and Hprt housekeeping genes with 5-month-old mice used as the reference; $\mathrm{n}=5-6$ mice per age group, except for the 5-month-old mice in (A) and (C) which had $\mathrm{n}=3$ and 2 , respectively, due to many samples being below the detection threshold. **p $<0.008$, *** $p<0.0005$, **** $p<$ 0.0001, significant difference between group with reference group, ANOVA with Fisher's LSD.
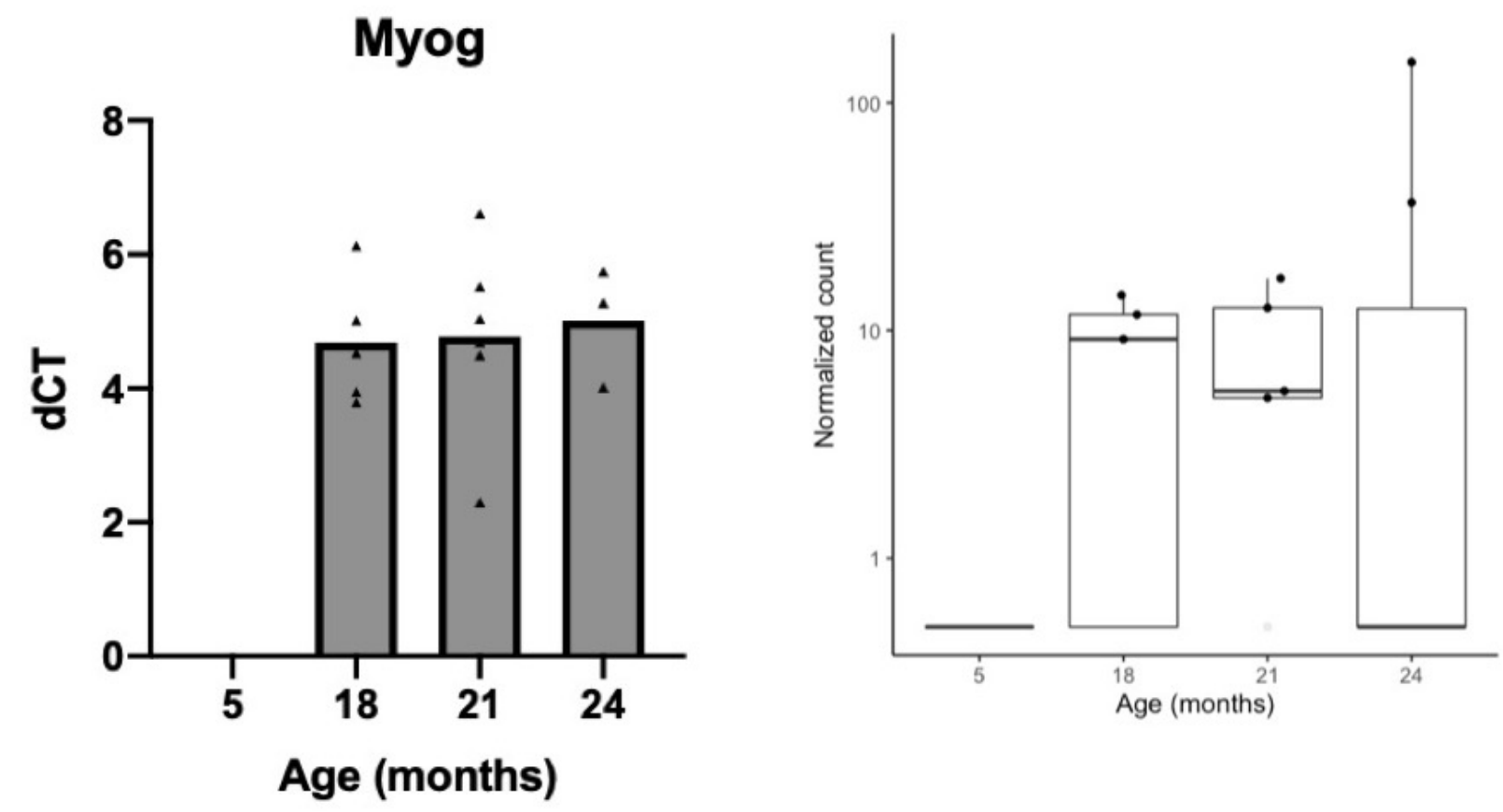

Figure 3. Myogenin (Myog) expression in sciatic nerve of mice aged 5, 18, 21, and 24 months. For the RT-PCR analysis, the number of samples for mice aged 5, 18, 21, and 24 months are $n=0$ (not detected), 5, 6, and 3, respectively. 


\subsection{Pattern identification and functional enrichment analyses of DEGs}

To investigate biologically relevant changes in gene expression across age groups, we analyzed all pair-wise comparisons of the normalized RNA-seq counts simultaneously using the Likelihood Ratio Test. This test identified 2,925 genes significant at FDR $<0.05$. We then identified clusters of genes within this list with similar expression patterns across age groups using the 'degPatterns' function in R. We identified seven clusters of genes containing 95-715 genes per cluster (Figure 4). Using GOrilla, we explored the biological relevance of these clusters using functional enrichment analysis assessing enrichment of clusters for GO Biological Process terms. The results of the functional enrichment analysis are shown in Figure 5.

Cluster 1 contained 476 genes and the gene list was significantly enriched with genes associated with extracellular matrix organization $\left(\right.$ FDR $q$-value $\left.=4.07 \times 10^{-5}\right)$ and extracellular structure organization $\left(\right.$ FDR $q$-value $\left.=4.77 \times 10^{-5}\right)$. Cluster 3 contained 715 genes which were significantly associated with 266 GO Biological Process terms at FDR $<0.05$. The top 31 terms are shown in Figure 5 including immune system process $\left(\right.$ FDR $q$-value $\left.=2.33 \times 10^{-19}\right)$, immune response $\left(\right.$ FDR $q$-value $\left.=8.04 \times 10^{-16}\right)$, and regulation of immune system process $($ FDR $q$-value $=$ $\left.5.74 \times 10^{-15}\right)$. Circadian regulation of gene expression (FDR $q$-value $\left.=0.0164\right)$, the cellular response to insulin stimulus $($ FDR $q$-value $=0.039)$, response to insulin $(\operatorname{FDR} q$-value $=0.026)$, and circadian rhythm (FDR $q$-value $=0.047)$ were significantly enriched GO terms of the 457 genes in Cluster 4. The 266 genes in Cluster 6 were significantly associated with 14 Biological Process GO terms including peptide biosynthetic process (FDR $q$-value $=1.07 \times 10^{-21}$ ), translation $\left(\right.$ FDR $q$-value $\left.=1.89 \times 10^{-21}\right)$, and peptide metabolic process $\left(\right.$ FDR $q$-value $\left.=1.24 \times 10^{-18}\right)$. The full results are available in Supplemental Spreadsheet 2. 

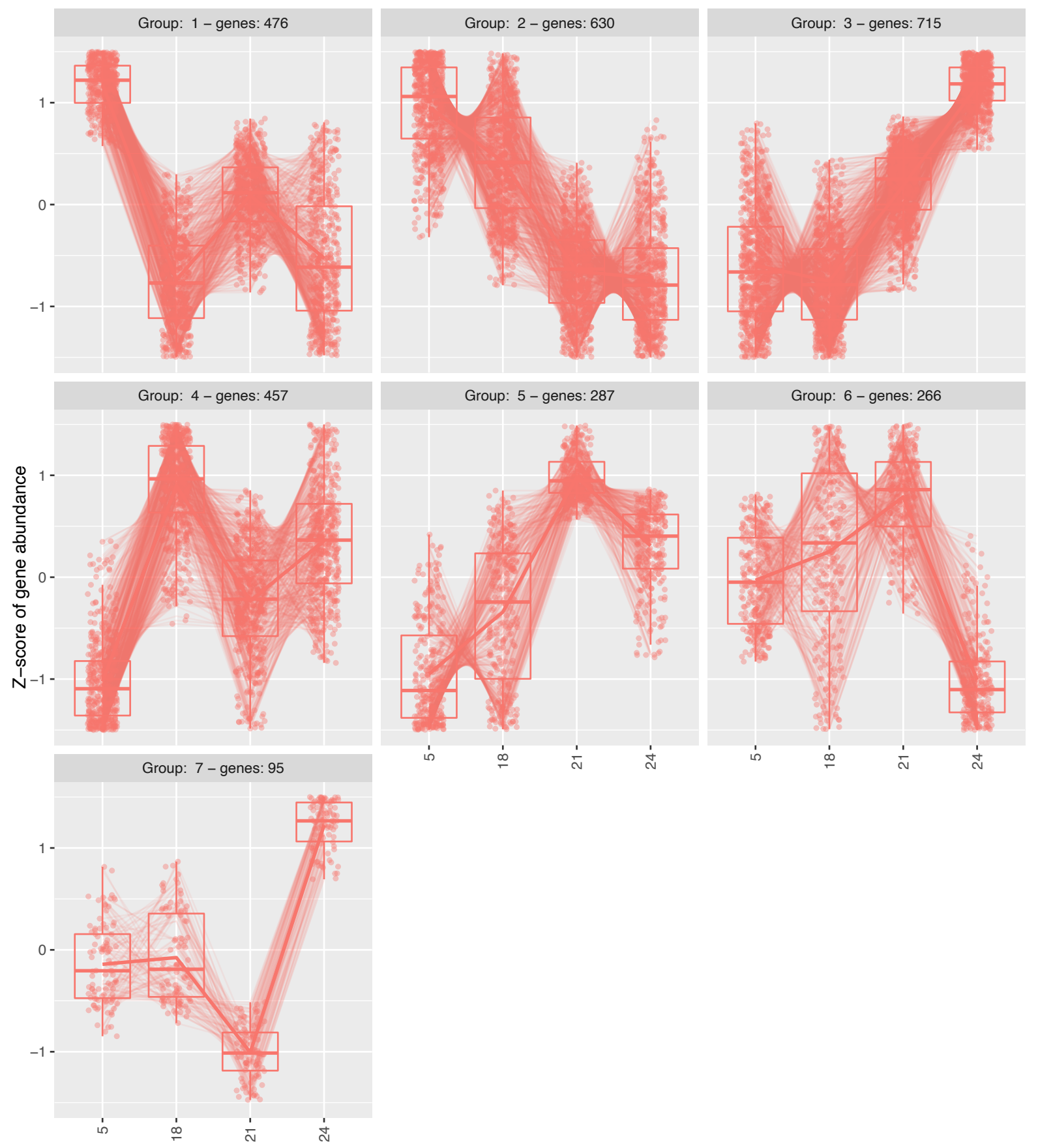

Figure 4. Clusters of genes with similar pattern of gene expression across age groups (labeled on the $\mathrm{x}$ axis). Identified from 2,925 genes significant in the Likelihood Ratio Test at FDR $<0.05$. 
bioRxiv preprint doi: https://doi.org/10.1101/2022.02.01.478571; this version posted February 3, 2022. The copyright holder for this preprint (which was not certified by peer review) is the author/funder, who has granted bioRxiv a license to display the preprint in perpetuity. It is made available under aCC-BY-NC-ND 4.0 International license.
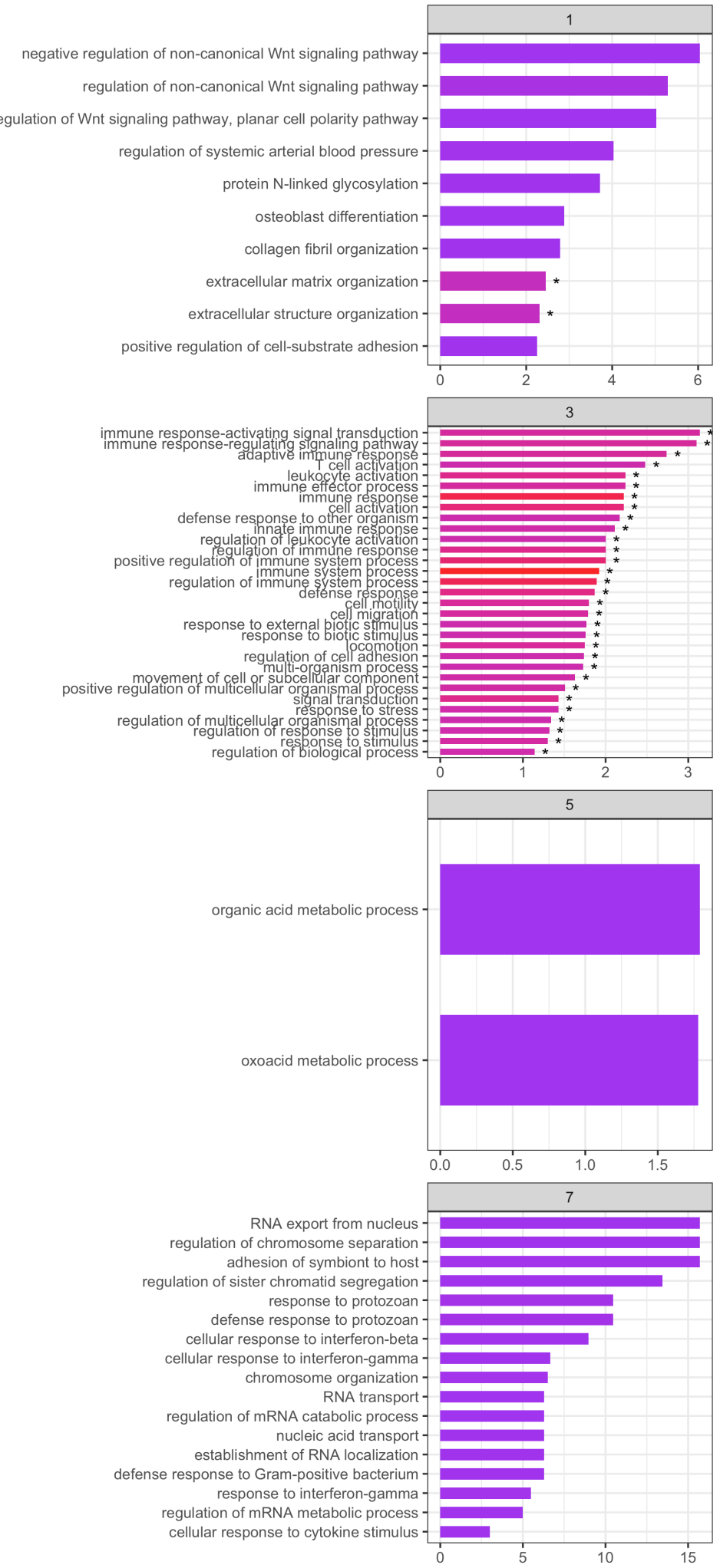

3

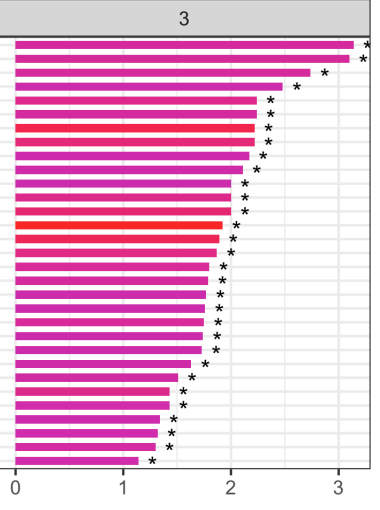

mitochondrial electron transport, cytochrome $\mathrm{c}$ to oxygen -

$$
\begin{array}{r}
\text { cytoplasmic translation - } \\
\text { cellular respiration } \\
\text { translation } \\
\text { peptide biosynthetic process - }
\end{array}
$$

peptide biosynthetic process
respiratory electron transport chain

electron transport chain
peptide metabolic process

amide biosynthetic process
cellular transition metal ion homeostasis

cellular amide metabolic process

ribonucleoprotein complex assembly

ribonucleoprotein complex subunit organization
cellular macromolecule biosynthetic process

rRNA metabolic process

macromolecule biosynthetic process

cellular protein-mpoining

cellular biosynthetic process
organic substance biosynthetic process
biosynthetic process

cellular nitrogen compound metabolic process

cellular protein metabolic process -
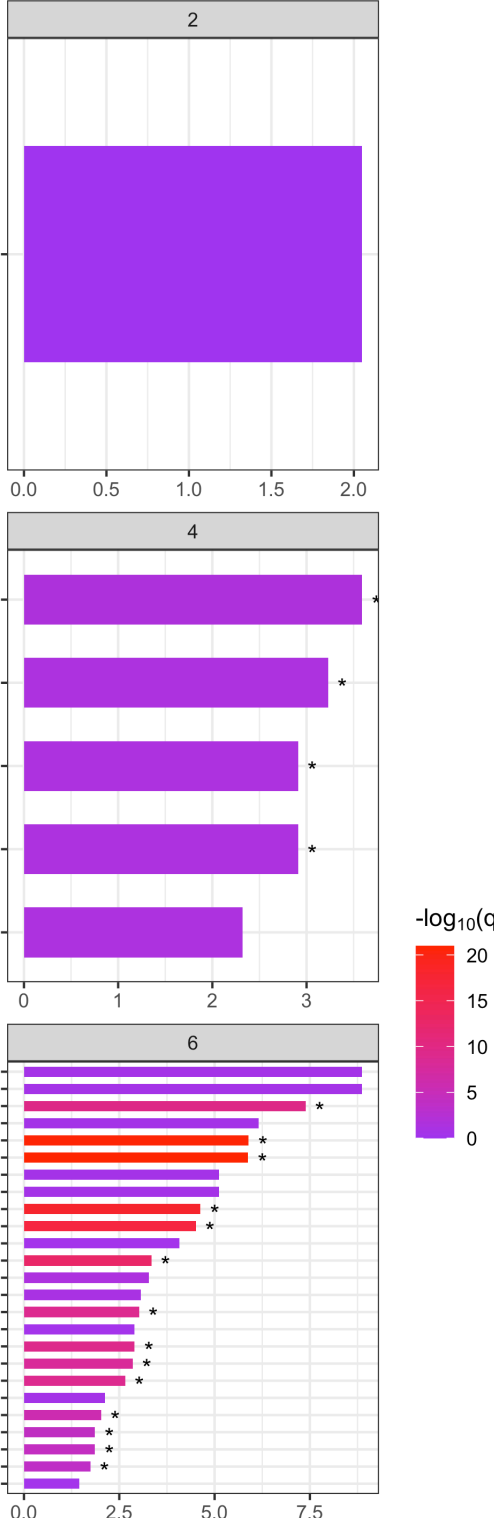

$-\log _{10}(\mathrm{q}-\mathrm{value})$

Enrichment

Figure 5. GO Biological Process functional enrichment analysis of the 7 clusters of gene expression, denoted in gray, identified by degPatterns. The Enrichment score is shown on the y 
bioRxiv preprint doi: https://doi.org/10.1101/2022.02.01.478571; this version posted February 3, 2022. The copyright holder for this preprint (which was not certified by peer review) is the author/funder, who has granted bioRxiv a license to display the preprint in perpetuity. It is made available under aCC-BY-NC-ND 4.0 International license.

axis, with bars colored according to $-\log _{10}(q$-value $)$ * GO biological process terms significantly enriched with target genes, FDR $q$-value $<0.05$. 


\section{Discussion}

Sarcopenia is the age-associated progressive decline in skeletal muscle mass and strength (Rolland et al. 2008; Landi et al. 1999; Rosenberg 1989). Such compromised muscle function restricts mobility and daily activity, greatly reducing the quality of life and causing a loss of independence among affected individuals. Low skeletal muscle mass or strength is also a frequent cause of disability in the elderly and is associated with many adverse outcomes including increased risk of falls and related fractures, morbidity, frailty, and mortality, making it a significant public health issue (Janssen et al. 2004; Metter et al. 2002; Abellan van Kan 2009).

Sarcopenia has long been considered a disease of skeletal muscle fibers only but accumulating evidence suggests that sarcopenia may originate in the nervous system. For example, electrophysiological examinations of patients have observed a reduction in motor unit numbers (Vandervoot and Symons 2001; Doherty et al. 1993) as well as an increase in the motor unit size (Tieland, Trouwborst, and Clark 2018; Lexell 1995), an indication of motor neuron death and distal motor neuropathy, respectively, resulting in decreased muscle strength. Thus, the neurological contribution to sarcopenia is thought to primarily occur through a loss of alpha motor neuron axons and the fiber type grouping that results from the concomitant remodeling of motor units: A decline in alpha motor neuron axons is observed in aging (Edström et al. 2007; McComas 1998) accompanied by changes in the motor neuron phenotype such as reduced dendritic tree size (Ramírez and Ulfhake 1992) and decreased synaptic input (Kullberg et al. 1998; Ramírez-León et al. 1999; Bergman and Ulfhake 2002). Surviving motor neurons compensate for the reduction in alpha motor neuron numbers by branching their axons to innervate the denervated muscle fibers (collateral sprouting), resulting in an increased number of muscle fibers per motor unit. However, it is unclear whether alteration of motor units is one of the first stages leading to sarcopenia.

To test our hypothesis that sarcopenia initiation and its early molecular programming could be detected by gene expression changes in nerves preceding sarcopenia development in muscle, we performed transcriptomic profiling of aging sciatic nerves in vivo. Signs of alterations in peripheral nerves prior to the clinical expression of sarcopenia is supported by a previous longitudinal study in C57BL/6J mice (aged between 4-24 months) that measured significant accumulation in the sciatic nerve of a series of proteins implicated in cytoskeleton dynamics/axonal transport and autophagic protein degradation pathways starting at 18 months 
(Krishnan et al. 2016), while in the same mice, loss of muscle mass and denervation at the NMJ is only clearly substantiated by 24 months of age (Barns et al. 2014; Chai et al. 2011). Another elegant study combining experiments in both rat and human tissues provided evidence that sarcopenia in vulnerable skeletal muscles of the lower limb (e.g., gastrocnemius) is tightly correlated with neuromuscular dysfunction (e.g., in the connected sciatic nerve), and that skeletal muscles resistant to sarcopenia (e.g., triceps brachii) are those with intact neuromuscular transmission (e.g., radial nerve) (Pannérec et al. 2016). In this study, while gene expression profiling in the muscles was studied at adult (8-10 months), early-sarcopenic (18-20 months), and confirmed sarcopenic (22-24 months) stages, in the linked peripheral nerves it was only examined in adult and old sarcopenic mice, limiting the opportunities to identify early molecular changes with a higher likelihood of driving the initiation and progression of sarcopenia.

The present study assessed gene expression in the sciatic nerve of mice aged 5, 18, 21, and 24 months using both untargeted RNA-seq and qRT-PCR, and measured expression of genes associated with muscle denervation in gastrocnemius muscle of the same mice using qRT-PCR. Altogether, we showed that expression of genes previously implicated in sarcopenia are differentially expressed $(|\mathrm{LFC}|>1.0, \mathrm{FDR}<0.05)$ in nerve as early as 18 months compared to healthy young adult controls, namely Myog (for review see Riuzzi et al. 2018) which was upregulated with a $\log _{2}$ fold change $\sim 19$ and Arntl (also known as Bmall, Kondratov et al. 2006) which showed a nearly four-fold decrease. Pathway enrichment analysis revealed that upregulated DEGs were borderline associated with the tight junction $(p=0.057)$ and AMPK signaling pathway $(p=0.08)$ while down-regulated DEGs were associated with biosynthesis and metabolic pathways. Both up- and down-regulated DEGs were associated with circadian rhythm, suggesting this is a particularly important pathway in aging sciatic nerve. We detected gene expression changes at 18 months, prior to the onset of sarcopenia, which we confirmed at 24 months by up-regulation of Chrng, Chrnd, Runxl, and Gadd45a in muscle by qRT-PCR, as upregulation of these genes has previously been demonstrated to coincide with sarcopenia onset (Barns et al. 2014).

To identify the earliest transcriptomic changes that could be occurring in sarcopenia, we closely examined gene expression in sciatic nerve of mice aged 18 months compared to mice aged 5 months. Mice aged 18- to 24-months are equivalent to 56- to 69-year old humans, and the presence of early signs of sarcopenia in C57BL/6J mice at $\sim 20$ months of age has been reported, 
demonstrated by significantly decreased grip strength, exercise endurance, muscle volume, and muscle mass of these mice compared to 10-week-old C57BL/6J mice (Kim and Hwang 2020). While most studies agree that 24- to 25-month-old mice represent a reliable animal model of sarcopenia, as both muscle mass and strength are affected at this stage and linked with NMJ loss (Barns et al. 2014), most studies utilizing the natural aging C57BL/6J mouse model of sarcopenia study mice older than 24 months of age (Xie et al. 2021), obscuring the study of molecular mechanisms early in the disease course. As early as 18 months, we detected upregulation of Myog in sciatic nerve which suggests disturbances in presynaptic terminals (NMJs) because expression of this gene increases strongly in denervated muscles. We also detected significant down-regulation of Arntl in 18-month-old mice. Mice deficient in brain and muscle Arntl have reduced lifespans and show symptoms of premature aging including sarcopenia (Kondratov et al. 2006). Thus, reduced Arntl expression in sciatic nerve at 18 months could indicate that pathways related to initiation of sarcopenia are unfolding at this time point.

Arntl is a core component of the circadian clock. We found dysregulation in other circadian clock genes, including period circadian regulator 2 and 3 (Per2, Per3) and Npas2. The importance of circadian rhythm maintenance for the structure, function, and metabolism of skeletal muscle is clear when observing the muscle phenotype in models of molecular clock disruption; cycle disruptions are linked to aging and the development of many chronic diseases including sarcopenia (Vitale et al. 2019). For example, circadian rhythm disruption was associated with an increased risk of sarcopenia in a population-based study in Korea which compared the risk of sarcopenia between night-shift workers and those who never worked night shifts (Choi et al. 2019). Our results suggest that impairment of circadian rhythm maintenance is an important factor in the development of sarcopenia that should be investigated further.

Additionally, our functional and pathway enrichment analysis of up-regulated DEGs identified the tight junction and AMPK signaling pathway as important pathways. The tight junction suggests the implication of the NMJ, while the appearance of the AMPK signaling pathway as an enriched pathway is a significant finding in the context of sarcopenia as this pathway plays a key role as a master regulator of cellular energy homeostasis (Mallick and Gupta 2021). The kinase is activated in response to stresses that deplete cellular ATP, such as low glucose or hypoxia. As a cellular energy sensor responding to low ATP levels, AMPK activation positively regulates signaling pathways that replenish cellular ATP supplies, including 
autophagy, and negatively regulates ATP-consuming biosynthetic processes such as protein synthesis. This agrees with our finding of down-regulation of biosynthetic and metabolic pathways. AMPK has also been implicated in a number of species as a critical modulator of aging through its interactions with mTOR and sirtuins, and thus this pathway could represent a relevant therapeutic target for the treatment of sarcopenia (Mallick and Gupta 2021).

We also performed functional enrichment analysis on clusters of genes with similar patterns of expression across age groups. We hypothesized that genes belonging to clusters 2 and 3, which showed progressive decreases and increases in expression over time, respectively, may be associated with natural aging. Indeed, these genes were related to regulation of transcription in agreement with previous reports of a systematic age-related decrease in regulation of transcription (Barns et al. 2014). These clusters were also associated with themes related to immune responses/responses to external stimuli, programmed cell death, and development and transcriptional regulation. Clusters 1 and 4 were of particular interest given their decrease and increase in expression at 18 months, respectively, which corresponds to the pre-onset of sarcopenia in C57BL/6J mice. Genes in cluster 1 were significantly associated with extracellular matrix organization and extracellular structure organization, suggestive of tissue remodeling with age. This is in agreement with previous reports in muscle of mice aged 24 months (Barns et al. 2014).

A limitation of this study is that we did not assess muscle strength/function or perform histology to validate the clinical and pathological onset and full development of sarcopenia and verify altered NMJ morphology and subsequent muscle denervation. However, these features of sarcopenia have previously been extensively characterized in the C57BL/6J mice we used for our study (Barns et al. 2014; Graber et al. 2015; Pannérec et al. 2016; Krishnan et al. 2016), and we confirmed that mice were sarcopenic by 24 months of age by replicating previous findings by Barns and colleagues demonstrating that expression of genes associated with NMJ denervation including Chrnd, Runxl, and Gadd45a are up-regulated in the muscle of mice aged 24 months. However, we did not detect significant up-regulation of Myog, although there was a nonsignificant increase in expression compared to expression in 5-month-old mice. Another limitation is that we performed RNA-seq on total RNA isolated from the entire sciatic nerve, as opposed to pure motor nerves. As is the case with many of the large nerves of the vertebrate nervous system, the sciatic nerve is a mixed-function nerve, meaning it is made up of the axons 
of both sensory and motor neurons. Therefore, in addition to motor neuron axons, we sequenced some sensory axons as well. Interestingly, the sympathetic nervous system has recently gained attention for its role in regulating skeletal muscle motor innervation as well as its potential implication in sarcopenia pathogenesis (Delbono et al. 2021). Glial cells are also in close contact with neurons within the sciatic nerve, and therefore in our study we measured some changes relevant to this other cellular compartment. Glial cells are instrumental in neural metabolism and regulation of neurotransmission; they were thus also proposed to potentially have a leading role in neuromuscular aging and sarcopenia (Kwan 2013). Future studies could use novel spatial imaging techniques such as the RNAScope ${ }^{\mathrm{TM}}$ to untangle the cellular origin of the key molecular changes we reported here.

In the current study, we investigated the mechanisms contributing to skeletal muscle weakness and atrophy during aging using an unbiased bioinformatics analysis. To our knowledge, this is the first report assessing the transcriptome of sciatic nerves in the context of sarcopenia using untargeted RNA-seq. The strengths of this study include the use of unbiased RNA-seq over microarrays, validation of our RNA-seq findings using qRT-PCR, and the use of multiple time points during the mouse life span with multiple $(n=5-6)$ biological replicates. Our results suggest that pathophysiological processes contributing to sarcopenia may be unfolding early in the nerve and suggest that increased understanding of the key mechanisms regulating skeletal muscle denervation at the NMJ could be critical to identifying novel treatments and biomarkers for sarcopenia in older adults.

\section{Acknowledgements}

This work was supported by the National Institutes of Health (NIH) National Institute on Aging (NIA) Grant (R21AG052011) awarded to D.B.R. and S.K., the National Institute of Environmental Health Sciences (NIEHS) Grant ES009089, and by the Ruth L. Kirschstein National Research Service Award Individual Predoctoral Fellowship (F31ES030973, awarded to N.C.). S.C., H.J.R., D.A., and A.M. are supported by the European Union's Horizon 2020 Research and Innovation Programme under the Marie Sklodowska-Curie grant agreement (No. 778003). This research was also funded in part through the National Cancer Institute (NCI) Cancer Center Support Grant (P30CA013696) and used the Genomics and High Throughput 
Screening Shared Resource. We thank Chaolin Zhang for his assistance in interpreting the RNAseq data.

\section{Conflict of Interest}

The authors have declared that they have no competing interests.

\section{Ethical Statement}

The present study was conducted in accordance with the Guiding Principles for the Care and Use of Laboratory Animals, as adopted by the IACUC of Columbia University (New York, New York, USA). 


\section{References}

Abellan van Kan, G. 2009. "Epidemiology and Consequences of Sarcopenia." The Journal of Nutrition, Health \& Aging 13 (8): 708-12. https://doi.org/10.1007/s12603-009-0201-z.

Andrews, Simon. 2010. "FastQC: A Quality Control Tool for High Throughput Sequence Data." 2010. https:/www.bioinformatics.babraham.ac.uk/projects/fastqc/.

Barns, Mitchell, Cedric Gondro, Ross L. Tellam, Hannah G. Radley-Crabb, Miranda D. Grounds, and Tea Shavlakadze. 2014. "Molecular Analyses Provide Insight into Mechanisms Underlying Sarcopenia and Myofibre Denervation in Old Skeletal Muscles of Mice." The International Journal of Biochemistry \& Cell Biology 53 (August): 17485. https://doi.org/10.1016/j.biocel.2014.04.025.

Bergman, Esbjörn, and Brun Ulfhake. 2002. "Evidence for Loss of Myelinated Input to the Spinal Cord in Senescent Rats." Neurobiology of Aging 23 (2): 271-86. https://doi.org/10.1016/s0197-4580(01)00274-3.

Chai, Ruth Jinfen, Jana Vukovic, Sarah Dunlop, Miranda D. Grounds, and Thea Shavlakadze. 2011. "Striking Denervation of Neuromuscular Junctions without Lumbar Motoneuron Loss in Geriatric Mouse Muscle." Edited by Stuart E. Dryer. PLoS ONE 6 (12): e28090. https://doi.org/10.1371/journal.pone.0028090.

Choi, Youn I, Dong Kyun Park, Jun-Won Chung, Kyoung Oh Kim, Kwang An Kwon, and Yoon Jae Kim. 2019. "Circadian Rhythm Disruption Is Associated with an Increased Risk of Sarcopenia: A Nationwide Population-Based Study in Korea." Scientific Reports 9 (1): 12015. https://doi.org/10.1038/s41598-019-48161-w.

Delbono, Osvaldo, Anna Carolina Zaia Rodrigues, Henry Jacob Bonilla, and Maria Laura Messi. 2021. "The Emerging Role of the Sympathetic Nervous System in Skeletal Muscle Motor Innervation and Sarcopenia." Ageing Research Reviews 67 (May): 101305. https://doi.org/10.1016/j.arr.2021.101305.

Dennis, Glynn, Brad T Sherman, Douglas A Hosack, Jun Yang, Wei Gao, H Clifford Lane, and Richard A Lempicki. 2003. "DAVID: Database for Annotation, Visualization, and Integrated Discovery." Genome Biology 4 (9). https://doi.org/10.1186/gb-2003-4-9-r60.

Dobin, Alexander, and Thomas R. Gingeras. 2015. "Mapping RNA-seq Reads with STAR." Current Protocols in Bioinformatics 51 (1). https://doi.org/10.1002/0471250953.bi1114s51.

Doherty, T. J., A. A. Vandervoort, A. W. Taylor, and W. F. Brown. 1993. "Effects of Motor Unit Losses on Strength in Older Men and Women." Journal of Applied Physiology (Bethesda, Md.: 1985) 74 (2): 868-74. https://doi.org/10.1152/jappl.1993.74.2.868.

Eden, Eran, Roy Navon, Israel Steinfeld, Doron Lipson, and Zohar Yakhini. 2009. "GOrilla: A Tool for Discovery and Visualization of Enriched GO Terms in Ranked Gene Lists." BMC Bioinformatics 10 (February): 48. https://doi.org/10.1186/1471-2105-10-48.

Edström, Erik, Mikael Altun, Esbjörn Bergman, Hans Johnson, Susanna Kullberg, Vania Ramírez-León, and Brun Ulfhake. 2007. "Factors Contributing to Neuromuscular Impairment and Sarcopenia during Aging." Physiology \& Behavior 92 (1-2): 129-35. https://doi.org/10.1016/j.physbeh.2007.05.040.

Ewels, Philip, Måns Magnusson, Sverker Lundin, and Max Käller. 2016. "MultiQC: Summarize Analysis Results for Multiple Tools and Samples in a Single Report." Bioinformatics 32 (19): 3047-48. https://doi.org/10.1093/bioinformatics/btw354.

Graber, Ted G., Jong-Hee Kim, Robert W. Grange, Linda K. McLoon, and LaDora V. Thompson. 2015. “C57BL/6 Life Span Study: Age-Related Declines in Muscle Power 
Production and Contractile Velocity." Age (Dordrecht, Netherlands) 37 (3): 9773. https://doi.org/10.1007/s11357-015-9773-1.

Huang, Da Wei, Brad T Sherman, and Richard A Lempicki. 2009. "Systematic and Integrative Analysis of Large Gene Lists Using DAVID Bioinformatics Resources." Nature Protocols 4 (1): 44-57. https://doi.org/10.1038/nprot.2008.211.

Huang, Da Wei, Brad T. Sherman, Qina Tan, Joseph Kir, David Liu, David Bryant, Yongjian Guo, et al. 2007. "DAVID Bioinformatics Resources: Expanded Annotation Database and Novel Algorithms to Better Extract Biology from Large Gene Lists." Nucleic Acids Research 35 (Web Server issue): W169-175. https://doi.org/10.1093/nar/gkm415.

Janssen, Ian, Richard N. Baumgartner, Robert Ross, Irwin H. Rosenberg, and Ronenn Roubenoff. 2004. "Skeletal Muscle Cutpoints Associated with Elevated Physical Disability Risk in Older Men and Women." American Journal of Epidemiology 159 (4): 413-21. https://doi.org/10.1093/aje/kwh058.

Kim, Changhee, and Jae-Kwan Hwang. 2020. “The 5,7-Dimethoxyflavone Suppresses Sarcopenia by Regulating Protein Turnover and Mitochondria Biogenesis-Related Pathways." Nutrients 12 (4): E1079. https://doi.org/10.3390/nu12041079.

Kondratov, Roman V., Anna A. Kondratova, Victoria Y. Gorbacheva, Olena V. Vykhovanets, and Marina P. Antoch. 2006. "Early Aging and Age-Related Pathologies in Mice Deficient in BMAL1, the Core Component of the Circadian Clock." Genes \& Development 20 (14): 1868-73. https://doi.org/10.1101/gad.1432206.

Krishnan, Vidya S., Zoe White, Chris D. McMahon, Stuart I. Hodgetts, Melinda Fitzgerald, Tea Shavlakadze, Alan R. Harvey, and Miranda D. Grounds. 2016. "A Neurogenic Perspective of Sarcopenia: Time Course Study of Sciatic Nerves From Aging Mice." Journal of Neuropathology \& Experimental Neurology 75 (5): 464-78. https://doi.org/10.1093/jnen/nlw019.

Kullberg, S., V. Ramírez-León, H. Johnson, and B. Ulfhake. 1998. "Decreased Axosomatic Input to Motoneurons and Astrogliosis in the Spinal Cord of Aged Rats." The Journals of Gerontology. Series A, Biological Sciences and Medical Sciences 53 (5): B369-379. https://doi.org/10.1093/gerona/53a.5.b369.

Kwan, Ping. 2013. "Sarcopenia: The Gliogenic Perspective.” Mechanisms of Ageing and Development 134 (9): 349-55. https://doi.org/10.1016/j.mad.2013.06.001.

Landi, Francesco, Giuseppe Zuccala, Giovanni Gambassi, Raffaele Antonelli Incalzi, Luca Manigrasso, Francesco Pagano, PierUgo Carbonin, and Roberto Bernabei. 1999. "Body Mass Index and Mortality Among Older People Living in the Community." Journal of the American Geriatrics Society 47 (9): 1072-76. https://doi.org/10.1111/j.15325415.1999.tb05229.x.

Lexell, J. 1995. "Human Aging, Muscle Mass, and Fiber Type Composition." The Journals of Gerontology. Series A, Biological Sciences and Medical Sciences 50 Spec No (November): 11-16. https://doi.org/10.1093/gerona/50a.special_issue.11.

Liao, Yang, Gordon K. Smyth, and Wei Shi. 2014. "FeatureCounts: An Efficient General Purpose Program for Assigning Sequence Reads to Genomic Features." Bioinformatics (Oxford, England) 30 (7): 923-30. https://doi.org/10.1093/bioinformatics/btt656.

Love, Michael I, Wolfgang Huber, and Simon Anders. 2014. "Moderated Estimation of Fold Change and Dispersion for RNA-Seq Data with DESeq2." Genome Biology 15 (12). https://doi.org/10.1186/s13059-014-0550-8. 
Mallick, Avijit, and Bhagwati P. Gupta. 2021. “AXIN-AMPK Signaling: Implications for Healthy Aging." F1000Research 10: 1259.

https://doi.org/10.12688/f1000research.74220.1.

McComas, Alan J. 1998. “1998 ISEK Congress Keynote Lecture.” Journal of Electromyography and Kinesiology 8 (6): 391-402. https://doi.org/10.1016/S1050-6411(98)00020-0.

Metter, E. Jeffrey, Laura A. Talbot, Matthew Schrager, and Robin Conwit. 2002. "Skeletal Muscle Strength as a Predictor of All-Cause Mortality in Healthy Men." The Journals of Gerontology. Series A, Biological Sciences and Medical Sciences 57 (10): B359-365. https://doi.org/10.1093/gerona/57.10.b359.

Pannérec, Alice, Margherita Springer, Eugenia Migliavacca, Alex Ireland, Mathew Piasecki, Sonia Karaz, Guillaume Jacot, et al. 2016. "A Robust Neuromuscular System Protects Rat and Human Skeletal Muscle from Sarcopenia.” Aging 8 (4): 712-29. https://doi.org/10.18632/aging.100926.

Ramírez, V., and B. Ulfhake. 1992. "Anatomy of Dendrites in Motoneurons Supplying the Intrinsic Muscles of the Foot Sole in the Aged Cat: Evidence for Dendritic Growth and Neo-Synaptogenesis." The Journal of Comparative Neurology 316 (1): 1-16. https://doi.org/10.1002/cne.903160102.

Ramírez-León, V., S. Kullberg, O. P. Hjelle, O. P. Ottersen, and B. Ulfhake. 1999. "Increased Glutathione Levels in Neurochemically Identified Fibre Systems in the Aged Rat Lumbar Motor Nuclei." The European Journal of Neuroscience 11 (8): 2935-48. https://doi.org/10.1046/j.1460-9568.1999.00710.x.

Riuzzi, Francesca, Guglielmo Sorci, Cataldo Arcuri, Ileana Giambanco, Ilaria Bellezza, Alba Minelli, and Rosario Donato. 2018. "Cellular and Molecular Mechanisms of Sarcopenia: The S100B Perspective: S100B and Sarcopenia." Journal of Cachexia, Sarcopenia and Muscle 9 (7): 1255-68. https://doi.org/10.1002/jcsm.12363.

Rolland, Y., S. Czerwinski, G. Abellan Van Kan, J. E. Morley, M. Cesari, G. Onder, J. Woo, et al. 2008. "Sarcopenia: Its Assessment, Etiology, Pathogenesis, Consequences and Future Perspectives." The Journal of Nutrition, Health \& Aging 12 (7): 433-50. https://doi.org/10.1007/bf02982704.

Rosenberg, Irwin H. 1989. "Summary Comments.” 50(Suppl): 1231S-3S.

Ryall, James G., Jonathan D. Schertzer, and Gordon S. Lynch. 2008. "Cellular and Molecular Mechanisms Underlying Age-Related Skeletal Muscle Wasting and Weakness." Biogerontology 9 (4): 213-28. https://doi.org/10.1007/s10522-008-9131-0.

Sherman, Brad T., Da Wei Huang, Qina Tan, Yongjian Guo, Stephan Bour, David Liu, Robert Stephens, Michael W. Baseler, H. Clifford Lane, and Richard A. Lempicki. 2007. "DAVID Knowledgebase: A Gene-Centered Database Integrating Heterogeneous Gene Annotation Resources to Facilitate High-Throughput Gene Functional Analysis.” BMC Bioinformatics 8 (November): 426. https://doi.org/10.1186/1471-2105-8-426.

Tieland, Michael, Inez Trouwborst, and Brian C. Clark. 2018. "Skeletal Muscle Performance and Ageing." Journal of Cachexia, Sarcopenia and Muscle 9 (1): 3-19. https://doi.org/10.1002/jcsm.12238.

Vandervoot, A. A., and T. B. Symons. 2001. "Functional and Metabolic Consequences of Sarcopenia." Canadian Journal of Applied Physiology = Revue Canadienne De Physiologie Appliquee 26 (1): 90-101. https://doi.org/10.1139/h01-007.

Vitale, Jacopo, Matteo Bonato, Antonio La Torre, and Giuseppe Banfi. 2019. "The Role of the Molecular Clock in Promoting Skeletal Muscle Growth and Protecting against 
Sarcopenia.” International Journal of Molecular Sciences 20 (17): 4318. https://doi.org/10.3390/ijms20174318.

Wang, Zhong, Mark Gerstein, and Michael Snyder. 2009. "RNA-Seq: A Revolutionary Tool for Transcriptomics." Nature Reviews. Genetics 10 (1): 57-63. https://doi.org/10.1038/nrg2484.

Xie, Wen-qing, Miao He, Deng-jie Yu, Yu-xiang Wu, Xiu-hua Wang, Shan Lv, Wen-feng Xiao, and Yu-sheng Li. 2021. "Mouse Models of Sarcopenia: Classification and Evaluation." Journal of Cachexia, Sarcopenia and Muscle 12 (3): 538-54. https://doi.org/10.1002/jcsm.12709.

Zhou, Jing, Zhiyin Liao, Jinliang Chen, Kexiang Zhao, and Qian Xiao. 2018. "Integrated Study on Comparative Transcriptome and Skeletal Muscle Function in Aged Rats." Mechanisms of Ageing and Development 169: 32-39.

https://doi.org/10.1016/j.mad.2018.01.001. 